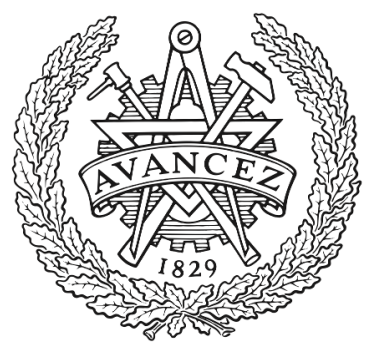

CHALMERS

UNIVERSITY OF TECHNOLOGY

\title{
Energy-Optimal Coordination of Autonomous Vehicles at Intersections
}

Downloaded from: https://research.chalmers.se, 2023-04-26 10:54 UTC

Citation for the original published paper (version of record):

Hult, R., Zanon, M., Gros, S. et al (2018). Energy-Optimal Coordination of Autonomous Vehicles at Intersections. 2018 European Control Conference (ECC): 602-607.

http://dx.doi.org/10.23919/ECC.2018.8550367

N.B. When citing this work, cite the original published paper. 


\title{
Energy-Optimal Coordination of Autonomous Vehicles at Intersections
}

\author{
Robert Hult, Mario Zanon, Sébastien Gros and Paolo Falcone
}

\begin{abstract}
The problem of coordinating automated vehicles at intersections is naturally posed within the optimal control framework, using objectives such as minimization of energy consumption. In this paper we extend previous work to include relevant nonlinearities in the vehicle models and propose a cost function that directly captures both energy consumption and travel time. The problem is a so-called Economic MPC (EMPC) problem, which entails both numerical and theoretical challenges. To address these issues, we propose to use a previously presented procedure to tune a MPC with a quadratic objective to approximate the EMPC. We evaluate the performance of both linear and nonlinear approximating MPC controllers in simulation. In particular, we demonstrate that a standard linear MPC can be tuned to so that the losses with respect to the EMPC is below $1 \%$.
\end{abstract}

\section{INTRODUCTION}

The introduction of cooperative, automated vehicles, enables new solutions for many traffic problems. For instance, at intersections, traffic lights and stop signs could be removed and the vehicles could rely on coordination algorithms to avoid collisions. Beyond increased safety, it is commonly argued that both energy efficiency and infrastructure utilization could be improved by such schemes.

Several works have presented schemes that utilize heuristics to solve this problem. For instance, [2] and [8] both propose schemes where an Intersection Manager allows or denies access to the intersection based on a predefined set of rules. Commonly, such heuristic schemes can guarantee safety, but have difficulties in claiming optimality and are usually hard to tune as their performance is implicitly defined by the specific rules.

Optimal control formulations, on the other hand, allow explicit performance objectives that are possible to tune to get a desired behavior. While it is commonly stated that minimization of energy usage and/or intersection throughput is the control objective, these criteria are commonly not explicitly included in the objective function [1], [4], [17]. Instead, for more convenient problem formulations, other quantities are optimized and it is commonly argued that this indirectly leads to the desired results. For instance, an objective function that penalizes the weighted sum of deviations from a desired speed and use of control effort is utilized in [1] and [6], where it is argued that this captures a trade-off between energy minimization and throughput. Another example is [17], where an objective is proposed that minimizes accelerations, which the authors argues minimizes engine transients and, therefore, fuel consumption.

The authors are with the department of Electrical Engineering at Chalmers Unicersity of Technology, Gothenburg, Sweden. \{hultr, zmario, grosse, pfalc\} @chalmers.se



Fig. 1: Schematic illustration of the scenarios considered in this paper. The arrows shows the fixed paths of the vehicles, and the red square illustrates the conflict zone where collisions can occur.

In this paper we utilize the problem formulation of [4], [6], [15], which we extend to include more realistic, nonlinear dynamics and an objective function that directly captures energy-usage and travel time delay. Due to the objective, the problem falls within the category of so-called Economic Model Predictive Control (EMPC), which entails both theoretical and numerical challenges. To address these challenges we apply the technique proposed in [13], where it is shown that under some conditions on local stability, a MPC with a positive definite quadratic cost can be designed which approximates the control law of the EMPC up to first order. In particular, we investigate two different formulations for the approximating positive definite MPC. In the first formulation, the nonlinear dynamics and constraints of the EMPC formulation are kept, and in the second linearizions of the dynamics and constraints are used. We demonstrate the differences between the EMPC and the two approximating controllers in simulation and compare them to a manually tuned NMPC with a quadratic tracking objective similar to that used in [6].

\section{MODELING}

We consider scenarios where $N_{a}$ electrical vehicles approach an intersection such that a collision might occur if no corrective action is taken. In this section, we model the scenario and state the optimal coordination problem.

\section{A. Intersection modeling and Collision avoidance}

We assume that all vehicles move along predefined and fixed paths and consider without loss of generality only simple intersections such as that shown in Fig. 1. Side collisions can only occur within the conflict zone $(\mathrm{CZ})$, illustrated in Fig. 1, and collision avoidance is ensured if no more than one vehicle is inside the $\mathrm{CZ}$ at any given time. 
We denote the index set $\mathbb{I}_{[a, b]}=\{a, a+1, \ldots, b-1, b\}$, for integers $a, b>a$, and let $p_{i}(t)$ denote the position of the center of vehicle $i \in \mathbb{I}_{\left[1, N_{a}\right]}$ along its path. Furthermore, we let $p_{i}^{\text {in }}$ and $p_{i}^{\text {out }}$ denote the beginning and end of the $\mathrm{CZ}$ on the path of vehicle $i$, as illustrated in Fig. 1 , and let $L_{i}$ be the vehicle length. We also let $t_{i}^{\text {in }}$ and $t_{i}^{\text {out }}$ be the times at which vehicle $i$ enters and exits the $\mathrm{CZ}$ respectively, which we define through the implicit relationships

$$
p_{i}\left(t_{i}^{\text {in }}\right)=p_{i}^{\text {in }}-L_{i} / 2 \quad \text { and } \quad p_{i}\left(t_{i}^{\text {out }}\right)=p_{i}^{\text {out }}+L_{i} / 2 .
$$

The side collision avoidance condition can thereafter be expressed as

$$
t_{i}^{\text {out }} \leq t_{j}^{\text {in }}
$$

when vehicles $i$ and $j$ are on different lanes and $i$ crosses the $\mathrm{CZ}$ before $j$.

\section{B. Vehicle Dynamics and Constraints}

Since all vehicles are assumed to move along fixed paths, we consider only the longitudinal vehicle dynamics

$$
\begin{aligned}
& \dot{p}_{i}(t)=v_{i}(t), \\
& \dot{v}_{i}(t)=\frac{1}{m_{i}}\left(F_{d, i}(t)-F_{b, i}(t)-F_{a, i}(t)-F_{r, i}(t)\right),
\end{aligned}
$$

where $m_{i}$ is the vehicle mass, $F_{d, i}$ is the force resulting from the electric motor torque, $F_{b, i}$ is the retarding force from the friction brakes, $F_{a, i}$ is the aerodynamic drag and $F_{r, i}$ the rolling resistance. In particular,

$$
F_{a, i}(t)=\frac{1}{2} \rho A_{i} C_{d, i} v_{i}^{2}(t), \quad F_{r, i}(t)=m_{i} g C_{r, i}
$$

where $\rho$ is the air density, $g$ the gravitational acceleration, $A_{i}$ the projected frontal area of the vehicle, $C_{d, i}$ the aerodynamic drag coefficient and $C_{r, i}$ the rolling resistance coefficient [3].

For simplicity, we assume that the transmission losses are negligible and that the propulsive force is proportional to the engine torque $T_{m, i}(t)$ through

$$
F_{d, i}(t)=\frac{M_{f, i} M_{i}}{r_{w, i}} T_{m, i}(t),
$$

where $r_{w, i}$ is the wheel radius, $M_{f, i}$ the final gear ratio and $M_{i}$ the transmission gear ratio, both constant.

As is common in modern electrical vehicles, we assume that the vehicles are equipped with AC traction motors. For a given motor speed $\omega_{m, i}(t)$, the motor is subject to the following limitations

$$
\begin{aligned}
& 0 \leq T_{m, i}(t) \leq \min \left(T_{m, i}^{\max }, P_{i}^{\max } / \omega_{m, i}(t)\right), \\
& 0 \leq \omega_{m, i}(t) \leq \omega_{m, i}^{\max }
\end{aligned}
$$

where $T_{i}^{\max }$ the maximum torque, $P^{\max }$ is the maximum power that can be supplied continuously, and $\omega_{m}^{\max }$ is the maximum motor speed [3]. Note that the motor speed relates to the vehicle speed through $v_{i}(t)=\frac{r_{w}}{M_{f} M} \omega_{m, i}(t)$.

While AC traction motors have internal dynamics, they are much faster than the dynamics of the vehicle, and are neglected for simplicity. We also neglect the dynamics of the friction brake system which similarly is much faster than the vehicle itself.

Consequently, the inputs of the electric vehicle are $u_{i}(t)=$ $\left(T_{m, i}(t), F_{b, i}(t)\right)$, and its states are $x_{i}(t)=\left(p_{i}(t), v_{i}(t)\right)$.

\section{Optimal Control formulation}

We formulate the coordination problem as an optimal control problem over a finite time-horizon $t_{f}$. In particular, we assume piece-wise constant inputs and discretize the dynamics (3) using numerical integration and multiple shooting with $N$ equally sized shooting intervals. The optimal intersection coordination problem can thereby be stated as the following nonlinear program (NLP):

$$
\begin{array}{ll}
\min _{W, T} & \sum_{i=1}^{N_{a}} J_{i}\left(w_{i}\right) \\
\text { s.t. } & \forall i \in \mathbb{I}_{\left[1, N_{a}\right]}: \\
& x_{i}(0)=\hat{x}_{i, 0}, \\
& x_{i, k+1}=F\left(x_{i, k}, u_{i, k}, \Delta t\right), \quad k \in \mathbb{I}_{[0, N-1]}, \\
& g\left(x_{i, k}, u_{i, k}\right) \geq 0, \\
& p_{i}^{d}\left(t_{i}^{\text {in }}, w_{i}\right)=p_{i}^{\text {in }}-L_{i} / 2, \\
& p_{i}^{d}\left(t_{i}^{\text {out }}, w_{i}\right)=p_{i}^{\text {out }}+L_{i} / 2, \\
& t_{i}^{\text {out }} \leq t_{j}^{\text {in }},
\end{array}
$$

where the control objective is on the form

$$
J_{i}\left(w_{i}\right)=V_{f, i}\left(x_{i, N}\right)+\sum_{i=0}^{N-1} \ell_{i}\left(x_{i, k}, u_{i, k}\right),
$$

$T=\left(t_{1}^{\text {in }}, t_{1}^{\text {out }}, \ldots, t_{N_{a}}^{\text {in }}, t_{N_{a}}^{\text {out }}\right), W=\left(w_{i}, \ldots, w_{N_{a}}\right), w_{i}=$ $\left(x_{i, 0}, u_{i, 0}, \ldots, x_{i, N-1}, u_{i, N-1}, x_{i, N}\right)$, and $\hat{x}_{i, 0}=\left(\hat{p}_{i, 0}, \hat{v}_{i, 0}\right)$ is the initial state of vehicle $i$. The function $F\left(x_{i, k}, u_{i, k}, \Delta t\right)$ denotes the integration of (3) over $t \in\left[t_{k}, t_{k}+t_{f} / N\right]$, starting from $x_{i, k}$ with input $u_{i}(t)=u_{i, k}$, whereas $g_{i}\left(x_{i, k}, u_{i, k}\right) \geq 0$ lumps together the motor constraints (6) and a bound on the friction brake force $F_{b, i}$. The function $p_{i}^{d}\left(t, w_{i}\right)$ is a continuous time representation of the position, defined as $p_{i}^{d}\left(t, w_{i}\right)=[1,0] F\left(x_{i, k}, u_{i, k}, \delta t\right), \delta t=t-k \Delta t$ and $k=$ $\lfloor t / \Delta t\rfloor$. Finally, the set $\mathcal{S}$ encodes the crossing order as the set of pair-wise precedence orders for access to the $\mathrm{CZ}$, which, for simplicity, is assumed given.

Closed-loop coordination is obtained through the iterative solution of the NLP (7) in a MPC fashion: Problem (7) is solved every $\Delta t$ and for all $i$ the first control of the optimal solution, $u_{i, 0}^{*}$ is applied to the vehicle.

Remark 1: As discussed in, e.g. [5], there are several practical issues relating to the solution of (7) in an on-line setting. These include what information the vehicles need to share, how wireless communication deficiencies should be handled, where computation takes place and what role, if any, roadside infrastructure plays in the process. Some of these issues are discussed in [6], [15] and [16]. In this paper, however, we do not consider such details in order to focus on the problem formulation. We therefore assume that a central coordinator is present in which all computations are performed without delay, and that communication between the vehicles and coordinator is both lossless and instantaneous. 


\section{Optimal Control Objective}

We consider two performance objectives: minimization of energy usage and minimization of travel-time. The two objectives are competing, as shorter travel-time requires higher average velocities and thereby higher average energy consumption. Simultaneous optimization of both therefore requires choices on trade-offs to be made by the control designer. In this section, we present and motivate one such choice, written on the form of (8).

\section{A. Selecting $\ell_{i}\left(x_{i, k}, u_{i, k}\right)$}

Dropping the vehicle index for brevity, the total consumed energy for a vehicle in the coordination problem is

$$
E(x(t), u(t))=\int_{0}^{t_{f}} P(x(t), u(t)) \mathrm{d} t,
$$

where $P(x(t), u(t))$ is the power supplied to the motor, defined as

$$
P(x(t), u(t))=\frac{\omega_{m}(t) T_{m}(t)}{\eta\left(\omega_{m}(t), T_{m}(t)\right)} .
$$

Here, $\eta(\cdot) \in[0,1]$ is a continuously differentiable function that describe the efficiency of the electric motor. Using numerical integration, (9) is discretized and written as $\sum_{k=0}^{N-1} \ell^{\mathrm{E}}\left(x_{k}, u_{k}\right)$, where

$$
\ell^{\mathrm{E}}\left(x_{k}, u_{k}\right)=\int_{k \Delta t}^{(k+1) \Delta t} P(x(t), u(t)) \mathrm{d} t .
$$

The energy-optimal solution to the coordination problem is to simply turn off the electric motor, whereby the vehicles would be decelerated to stand-still by the resistive forces. The main competing objective in the intersection problem is to induce as small travel delay as possible, i.e., to maximize the average velocity, which can be formulated using the stage cost

$$
\ell^{\mathrm{v}}\left(x_{k}, u_{k}\right)=\frac{1}{\Delta t} \int_{k \Delta t}^{(k+1) \Delta t} v(t) \mathrm{d} t .
$$

A natural stage cost for (8) is obtained by scalarization of the multi-objective problem as

$$
\ell_{\alpha}^{\mathrm{E}, \mathrm{v}}\left(x_{k}, u_{k}\right)=\ell^{\mathrm{E}}\left(x_{k}, u_{k}\right)-\alpha \ell^{\mathrm{v}}\left(x_{k}, u_{k}\right),
$$

where $\alpha>0$ is a trade-off parameter. Introducing the steadystate optimization problem,

$$
\min _{x, u} \quad \ell_{\alpha}^{\mathrm{E}, \mathrm{v}}(x, u) \text { s.t. } v=F_{v}(v, u), g(x, u) \geq 0,
$$

where $F_{v}(v, u)$ is the integration of (3b), we note that the value of $\alpha$ decides the optimal steady-state velocity $v_{\mathrm{s}}$.

Selection of $\alpha$ : For tuning purposes, it is easier to choose $v_{\mathrm{s}}$ than $\alpha$. To this end, we introduce the average-velocity constrained steady state problem

$$
\begin{array}{ll}
\min _{x, u} & \ell^{\mathrm{E}}(x, u) \\
\text { s.t. } & v=F_{v}(v, u), g(x, u) \geq 0, \ell^{\mathrm{v}}(x, u)=v_{\mathrm{r}},
\end{array}
$$

where $v_{\mathrm{r}}$ is a specified average velocity. Denoting the optimal Lagrange multiplier of the constraint $\ell^{\mathrm{v}}(x, u)-v_{\mathrm{r}}=0$ as $\nu_{\mathrm{r}}$, we note that the solution of (15) is a local solution to (14) when $\alpha=\nu_{\mathrm{r}}$. That is, when $\alpha=\nu_{\mathrm{r}}$, we impose the specified steady-state velocity $v_{\mathrm{s}}=v_{\mathrm{r}}$.

\section{B. Selecting $V_{f, i}\left(x_{i, N}\right)$}

We note that the gradient of $\ell_{\alpha}^{\mathrm{E}, \mathrm{v}}(x, u)$ can be non-zero at the steady state $\left(x_{\mathrm{s}}, u_{\mathrm{u}}\right)$. As shown in [11], when such objective functions are used in MPC, asymptotic stabilization to the steady state can be ensured by introducing a linear penalty term in the terminal cost $V_{f}\left(x_{N}\right)$. In particular, denoting the optimal Lagrange multiplier of the constraint $v_{\mathrm{s}}-F_{v}\left(v_{s}, u_{s}\right)=0$ in (14) as $\lambda_{\mathrm{s}}$, this is in our case ensured through the terminal penalty term $\lambda_{\mathrm{s}} v_{N}$.

Finally, to account for the finite horizon, we introduce a quadratic terminal cost penalty so that

$$
V_{f}\left(x_{N}\right)=\frac{1}{2}\left(v_{N}-v_{\mathrm{s}}\right)^{2} P+\lambda_{\mathrm{s}} v_{N},
$$

where we detail the selection of $P$ in Remark 2 of Section IV.

With this objective function, the coordination problem is a so-called Economic MPC (EMPC) problem. In the next section, we detail the application of a recently developed method for design of tracking MPC controllers which approximate the EMPC.

\section{EMPC AND LOCALLY EQUIVALENT TRACKING MPC}

Standard MPC schemes, also referred to as tracking MPC (TMPC), rely on positive definite (typically quadratic) functions $\ell(\cdot), V_{f}(\cdot)$ which penalize deviations from a given reference. EMPC on the other hand, directly optimizes more generic criteria, with $\ell(\cdot), V_{f}(\cdot)$ possibly indefinite.

While EMPC is expected to deliver better performance than TMPC in terms of a specific performance criteria, severe challenges arise regarding both the deployability of efficient numerical algorithms and its stability guarantees. In order to overcome these difficulties a procedure is proposed in [13] with which a TMPC can be tuned to approximate the EMPC control-law to first order, provided that the EMPC is stabilizing.

Next, we first briefly sketch how a quadratic tracking objective can be designed such that the Tracking MPC control law is a first-order approximation of the economic MPC control law and then discuss the method's application to the coordination problem.

\section{A. Locally Equivalent Tracking MPC}

For a discrete-time dynamical system $f(x, u)$, constrained by $h(x, u) \geq 0$ and with stage cost $\ell(x, u)$, we define the steady-state optimization problem as

$$
\min _{x, u} \ell(x, u) \quad \text { s.t. } x-f(x, u)=0, h(x, u) \geq 0,
$$

with primal solution $x_{\mathrm{s}}, u_{\mathrm{s}}$, dual solution $\lambda_{\mathrm{s}}, \mu_{\mathrm{s}}$, and Lagrange function $\mathcal{L}(x, u, \lambda, \mu)=\ell(x, u)-\lambda^{\top}(x-f(x, u))-$ $\mu^{\top} h(x, u)$. Moreover, we define

$$
\begin{aligned}
& A_{\mathrm{s}}:=\nabla_{x} f\left(x_{\mathrm{s}}, u_{\mathrm{s}}\right), \quad B_{\mathrm{s}}:=\nabla_{u} f\left(x_{\mathrm{s}}, u_{\mathrm{s}}\right), \\
& G_{\mathrm{s}}:=\nabla_{(x, u)} h\left(x_{\mathrm{s}}, u_{\mathrm{s}}\right), \quad H:=\nabla_{(x, u)}^{2} \mathcal{L}\left(x_{\mathrm{s}}, u_{\mathrm{s}}, \lambda_{\mathrm{s}}, \mu_{\mathrm{s}}\right) .
\end{aligned}
$$

In order to compute the quadratic term of the tracking cost, one needs to solve the following semi-definite program 


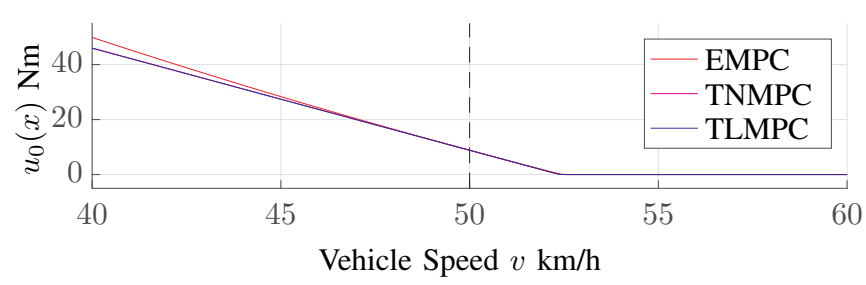

Fig. 2: Plot of the motor torque feedback law of the EMPC, TNMPC and TLMPC for a range of velocity values for one electric vehicle and $v^{\mathrm{r}}=50 \mathrm{~km} / \mathrm{h}$. Note the similarity of the control-laws close to the steady state $v_{\mathrm{s}}=v^{\mathrm{r}}$, illustrating the first order equivalence. Parameters as given in Section V.

(SDP) [13]:

$$
\begin{aligned}
\min _{\Pi, \Gamma, \beta, \zeta} & \gamma \beta-\zeta+\rho\|\Gamma\| \\
\text { s.t. } & \zeta I \preceq \underbrace{H+\mathcal{H}(\Pi)+G_{\mathrm{s}, \mathrm{A}}^{\top} \Gamma G_{\mathrm{s}, \mathrm{A}}}_{\tilde{H}} \preceq \beta I,
\end{aligned}
$$

where $\gamma$ and $\rho$ are tuning parameters, $G_{\mathrm{s}, \mathrm{A}}$ is obtained from $G_{\mathrm{S}}$ by selecting the rows corresponding to the strictly active inequality constraints and

$$
\mathcal{H}(\Pi)=\left[\begin{array}{cc}
A_{\mathrm{s}}^{\top} \Pi A_{\mathrm{s}}-\Pi & A_{\mathrm{s}}^{\top} \Pi B_{\mathrm{s}} \\
B_{\mathrm{s}}^{\top} \Pi A_{\mathrm{s}} & B_{\mathrm{s}}^{\top} \Pi B_{\mathrm{s}}
\end{array}\right] .
$$

If only linear inequality constraints are active ${ }^{1}$ at the optimal steady state, the stage cost is given by

$\ell^{Q}(x, u)=\frac{1}{2}\left[\begin{array}{l}x-x_{\mathrm{s}} \\ u-u_{\mathrm{s}}\end{array}\right]^{\top} \tilde{H}\left[\begin{array}{l}x-x_{\mathrm{s}} \\ u-u_{\mathrm{s}}\end{array}\right]+\nabla \ell(x, u)^{\top}\left[\begin{array}{l}x-x_{\mathrm{s}} \\ u-u_{\mathrm{s}}\end{array}\right]$,

with $\tilde{H}$ as in (18b). By solving (18), curvature is thus added to $H$ such that the resulting matrix $\tilde{H}$ is positive definite, whereby $\ell^{Q}(x, u)$ is convex. The quadratic stage cost can then be used both in tracking nonlinear MPC (TNMPC) and in tracking linear MPC (TLMPC). The feedback control law of both schemes will have the property of being a first-order approximation of the EMPC feedback control law. For more details on this topic, we refer the reader to [13], [12].

\section{B. Application to the Intersection Coordination Problem}

The intersection coordination problem (7) consists of a collection of sub-systems that are coupled while $p<p^{\text {in }}$, but decoupled when $p>p^{\text {out }}$. In particular, when active, the coupling constraints force the vehicles to change velocity. Unless no coupling constraints are active, or $p>0$ for all vehicles, there is therefore no notion of a steady state solution to (7). Instead, we consider the optimal control problem for one vehicle :

$$
\min _{w_{i}} J_{i}\left(w_{i}\right) \text { s.t. } \quad(7 \mathrm{~b}),(7 \mathrm{c}),(7 \mathrm{~d}),
$$

for which (14) is the corresponding steady state problem.

The EMPC based on (20) can now be approximated using the procedure of [13] based on (14). $J_{i}^{Q}\left(w_{i}\right)=$ $V_{f, i}^{Q}\left(x_{i, N}\right)+\sum_{k=0}^{N-1} \ell^{Q}\left(x_{i, k}, u_{i, k}\right)$ The first order equivalence

\footnotetext{
${ }^{1}$ Note that the case of active nonlinear inequality constraints can be easily handled in this formalism, but is omitted for brevity.
}

to the resulting tuned TNMPC and TLMPC controllers is illustrated Figure 2.

The couplings in the coordination problem can be seen as perturbations to the individual vehicles, forcing them to deviate from their desired steady state. We therefore propose to use the sum of the quadratic approximations of the vehicles in (7a) to approximate the coordination EMPC. We are interested in examining both the TNMPC and TLMPC case. The latter is particularly important, as it allows the application of the efficient distributed algorithms presented in [6], [15] and [7] to be used.

Remark 2: In the EMPC case, we choose the quadratic penalty weight $P$ as the cost-to-go associated with the LQR computed for $\left(A_{s}, B_{s}, H\right)$, where $\left(A_{s}, B_{s}, H\right)$ are obtained from (14) and the position is eliminated from the state vector. For the TNMPC and TLMPC, we instead use the terminal penalty $V_{f}^{Q}\left(x_{N}\right)=\frac{1}{2}\left(v_{N}-v_{\mathrm{s}}\right)^{2} \tilde{P}$, where $\tilde{P}$ is the cost-to-go associated with the LQR computed for $\left(A_{s}, B_{s}, \tilde{H}\right)$.

\section{NUMERICAL RESUlts}

In this section, we present simulation results where we compare the performance of the EMPC based on (7) to the tuned TNMPC and TLMPC. We also provide a comparison with an MPC that utilize a "standard" tracking objective similar to that of e.g. [6], [14]:

$$
\begin{aligned}
\ell^{q}(x, u)=\frac{1}{2}\left(\left(v-v_{\mathrm{s}}\right)^{2} Q_{v}+\right. & \left(T_{m}-T_{m, \mathrm{~s}}\right)^{2} R_{T_{m}} \\
& \left.+\left(F_{b}-F_{b, \mathrm{~s}}\right)^{2} R_{F_{b}}\right),
\end{aligned}
$$

which we denote the Standard MPC (SMPC). For simplicity, we let the weights be $R_{F_{b}}=\left(1 / F_{b}^{\max }\right)^{2}, R_{T_{m}}=$ $\left(1 / T^{\max }\right)^{2}$ and $Q_{v}=\left(1 / v^{\mathrm{r}}\right)^{2}$.

Vehicle Parameters: We consider scenarios with homogeneous vehicles, where the parameters of (3) are chosen in the range of values for a passenger vehicle, as summarized in Table I. Furthermore, we have $\rho=1.225, g=9.81, p^{\text {in }}=0$, $p^{\text {out }}=10.7$.

\begin{tabular}{|l|ccccccc|}
\hline Parameter & $L$ & $m$ & $A$ & $r_{w}$ & $M_{f} M$ & $C_{d}$ & $C_{r r}$ \\
\hline Value & 4.8 & 1700 & 2.3 & 0.32 & 7.9 & 0.35 & 0.015 \\
\hline
\end{tabular}

TABLE I: Vehicle parameters. $L, m, A, r_{w}$ are given in SI-units, and $M_{f} M, C_{d}$ and $C_{r r}$ are unitless.

We use the approximate electric motor efficiency map $\eta\left(T_{m}, \omega_{m}\right)$ of [9], where $\eta\left(T_{m}, \omega_{m}\right)=\frac{T_{m} \omega_{m}}{T_{m} \omega_{m}+P_{\text {loss }}\left(T_{m}, \omega_{m}\right)}$, with $P_{\text {loss }}\left(T_{m}, \omega_{m}\right)=c_{0}+c_{1} \omega_{m}+c_{2} \omega_{m} T_{m}+c_{3} \omega_{m}^{2}$, and coefficients $c_{i}$ obtained from a fit to empirical data. However, to better capture the performance of a passenger vehicle traction motor, $\eta\left(T_{m}, \omega_{m}\right)$ is scaled so that $P^{\max }=80$ $\mathrm{kW}, T^{\max }=280 \mathrm{Nm}$ and $\omega_{m}^{\max }=10000 \mathrm{rpm}$. Finally, the maximum friction brake force is $F_{b}^{\max }=10 \mathrm{kN}$.

We note that the choice of $\eta\left(T_{m}, \omega_{m}\right)$ is non-restrictive, and the formalism is capable of using other representations as well. 
Simulation: We apply the different controllers to a simulation of the vehicles using the nonlinear dynamics (3), which we integrate using Explicit Runge-Kutta-4 integrators (ERK4) from the ACADO toolkit [10]. For the EMPC, TNMPC and SMPC, which rely nonlinear prediction models, we use ERK4 integrators also inside the optimization problem (7). Finally, we set $\Delta t=0.1, N=100$.

\section{A. Simulation Results}

We consider an example scenario where 3 vehicles are starting $100 \mathrm{~m}$ before the intersection at $\hat{v}_{0, i}=v^{\mathrm{r}}=70$ $\mathrm{km} / \mathrm{h}$, i.e. all vehicles will reach the intersection simultaneously if no action is taken. The closed-loop state trajectories are given in Fig. 3, while a plot of the corresponding motor torque and speed is presented in Fig. 4. As is seen in the figures, the TNMPC and TLMPC produce a result which is very similar to the EMPC, while the SMPC differs significantly. In particular, the EMPC, TNMPC and TLMPC only utilize the friction brakes to avoid collisions, and do so during a very short time. The SMPC, on the other hand, uses the friction brakes both to avoid collisions and to reach $v^{\mathrm{r}}$, and applies it over a longer period of time. Instead, the first three controllers only accelerates the first and second vehicle and lets the last vehicle reduce its speed using the airdrag and rolling resistance alone. While initially more energy thereby is injected into the system than in the SMPC case, less energy is wasted by not using the friction brakes and less external energy needs to be supplied after the intersection. This behavior is illustrated in Fig. 5, which shows the development of the closed loop systems cumulative cost $J^{\mathrm{cl}}(K)=\sum_{k=0}^{K} \sum_{i=1}^{3} \ell_{i}^{\mathrm{E}, \mathrm{v}^{\mathrm{r}}}\left(x_{k}, u_{k}\right)$

It is clear that while the EMPC incurs a higher momentary cost in the beginning of the simulation compared to the SMPC, the former outperforms the latter in the long run. After 60 seconds of simulation, the difference between the EMPC and the two tuned tracking controllers is less than $1 \%$, while the difference to the SMPC is close to $20 \%$.

Limitations of the approximating controllers: While the scenario reported in Fig. 3 shows that both the TNMPC and TLMPC perform similarly to the EMPC, this is not necessarily the case in scenarios where the vehicles are operated far from the steady state. As an example, consider the motor torque-speed plot in Fig. 6, which is taken from a scenario where three vehicles are initialized at $70 \mathrm{~km} / \mathrm{h}$ 50 meters from the intersection. To avoid collision, all vehicles are forced to perform extreme maneuvers and deviate significantly from the steady state. However, the TLMPC fails to utilize the full control authority, and saturates the input at the linearized torque constraint (6) for the first two vehicles. Moreover, for the last vehicle, both the TLMPC and the TNMPC differs more significantly from the EMPC, which is likely due to a poor approximation of the objective function in that region of the $T_{m}-\omega_{m}$-space.

However, in a practical setting, the coordination controller would be applied when the vehicles are still far from the intersection, close to steady state conditions. In such cases, only small changes in velocity are required to avoid
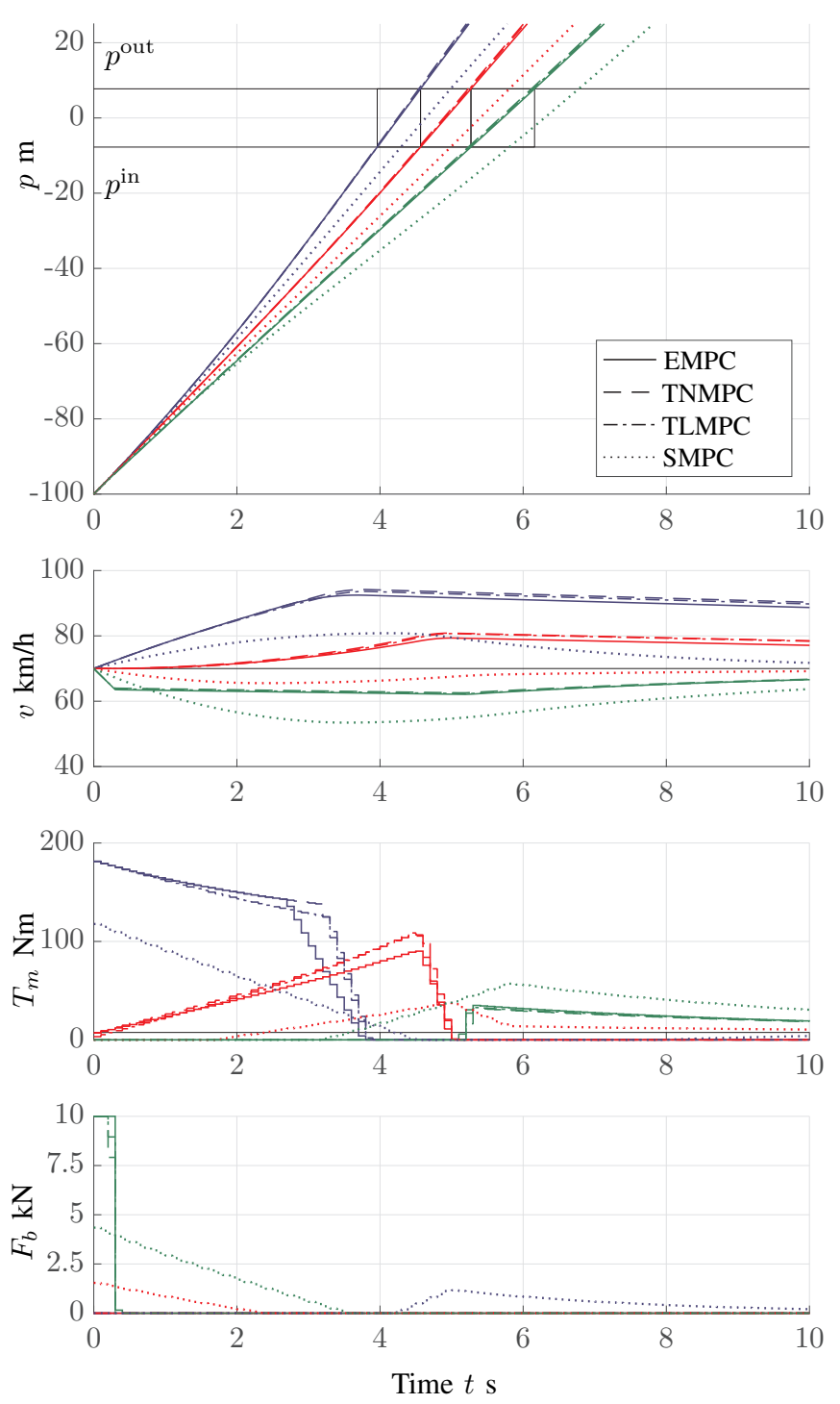

Fig. 3: Example scenario with three vehicles starting at $p=$ $-100 \mathrm{~m}$. In the topmost plot, the horizontal lines demarcate the intersection, whereas the vertical lines show $t_{i}^{\text {in }}, t_{i}^{\text {out }}, \forall i$. In all plots, the colors differentiates between vehicles and the line-styles between the controllers. In the three lower plots, the solid black lines are the solution to the steady state problem (14).

collisions, and the problem is likely avoided during normal operations.

Limitations of the chosen objective : While the stage cost (13) encodes a trade-off between energy consumption and traffic delay in an intuitive manner it has some drawbacks. Consider for instance the situation illustrated in Fig. 7 where the EMPC is used from a velocity significantly lower than $v^{\mathrm{r}}$. In this case, the controller applies high levels of motor torque to reduce the difference which cause initial accelerations of above $3.5 \mathrm{~m} / \mathrm{s}^{2}$. This would cause both passenger discomfort and unnecessary mechanical wear. Inclusion of additional objectives, e.g., comfort criteria, to the problem is therefore desirable. 


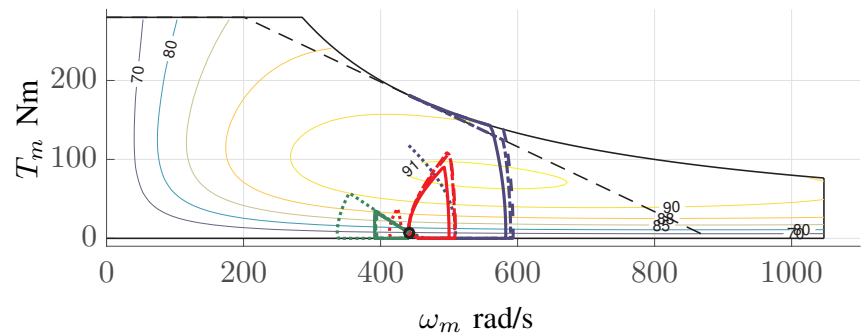

Fig. 4: Motor speed-torque trajectories corresponding to Fig. 3 superimposed on contours of $\eta\left(\omega_{m}, T_{m}\right)$ and the motor constraints (6). The black dot is the solution to the steady-state problem (14), and the dashed black line is the linearization of (6) around the steady-state solution, relevant for the TLMPC.

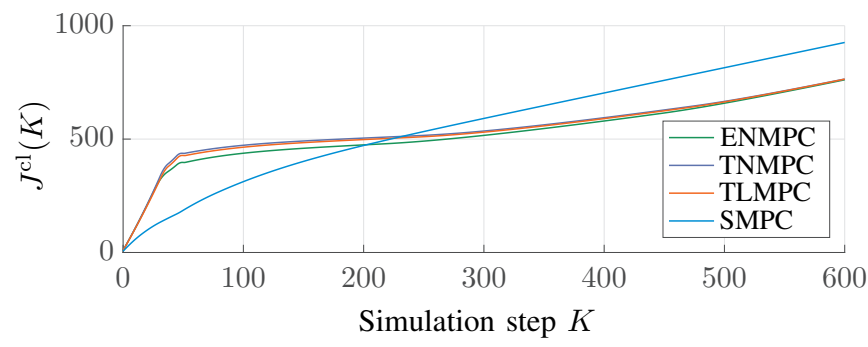

Fig. 5: Development of the cumulative cost $J^{\mathrm{cl}}(K)$

\section{CONCLUSION}

In this paper, we have extended earlier work on intersection coordination to include more accurate, nonlinear vehicle models and an economic objective function. We have detailed the application of a method for automatic tuning of both linear and nonlinear TMPC to approximate the EMPC, and demonstrated their performance through simulation.

We have showed that the approximating TMPC controllers provide a good alternative to EMPC for the intersection coordination problem and does not significantly degrade performance. The exception is for initial configurations that force some vehicles to deviate too far from their economic steady state. We stress that the performance of the TLMPC is particularly important, as it allows previous results on linear MPC based intersection coordination to be applied while retaining good economic performance. In particular, coordination based on linear MPC has been validated on real vehicles [16], implying the practical relevance of the TLMPC approximation. In future work, we intend to formulate an objective function that includes additional criteria, e.g., passenger comfort, and offers more control of the transient behavior of the vehicles.

\section{REFERENCES}

[1] G. R. de Campos, P. Falcone, H. Wymeersch, R. Hult, and J. Sjöberg. Cooperative receding horizon conflict resolution at traffic intersections. In 53rd CDC, pages 2932-2937, Dec 2014.

[2] K. Dresner and P. Stone. A Multiagent Approach to Autonomous Intersection Management. Journal of Artificial Intelligence Research, 31(1):591-656, March 2008.

[3] L. Guzzella and A. Sciarretta. Vehicle Propulsion Systems: Introduction to Modeling and Optimization. 2005.

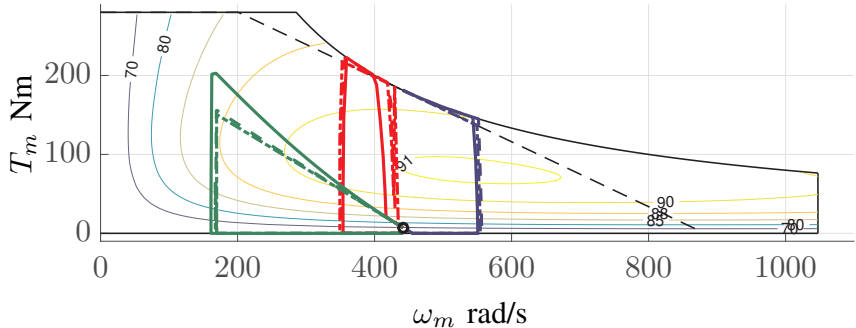

Fig. 6: Motor speed-torque trajectories of a more demanding scenario where the crossing order is red, yellow, green. Plot markers, colors and line-styles as in Fig. 4. Notice that the EMPC, TNMPC and TLMPC controllers now exhibits a larger difference.

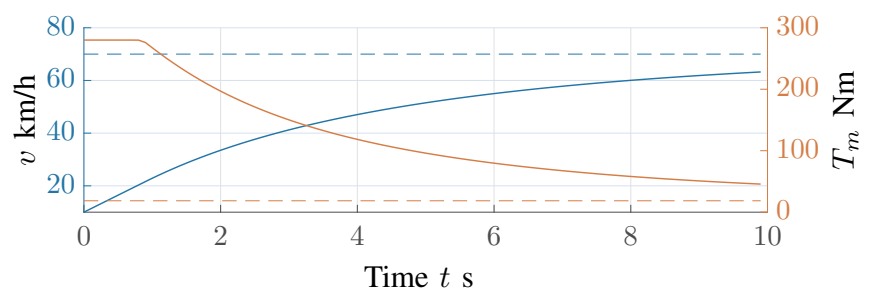

Fig. 7: Illustration of the transient behavior of the EMPC from an initial condition far from the steady state. Dashed lines are steady state values.

[4] R. Hult, G. R. de Campos, P. Falcone, and H. Wymeersch. An approximate solution to the optimal coordination problem for autonomous vehicles at intersections. In American Control Conference (ACC), 2015, pages 763-768, July 2015.

[5] R. Hult, G. R. de Campos, E. Steinmetz, L. Hammarstrand, P. Falcone, and $\mathrm{H}$. Wymeersch. Coordination of cooperative autonomous vehicles: Toward safer and more efficient road transportation. IEEE Signal Processing Magazine, 33(6):74-84, Nov 2016.

[6] R. Hult, M. Zanon, S. Gros, and P. Falcone. Primal Decomposition of the Optimal Coordination of Vehicles at Traffic Intersections. In Proceedings of the Conference on Decision and Control, 2016.

[7] Y. Jiang, M. Zanon, R. Hult, and B. Houska. Distributed algorithm for optimal vehicle coordination at traffic intersections. In Proceedings of the 20th IFAC World Congress, France, pages 12082-12087, 2017.

[8] H. Kowshik, D. Caveney, and P. R. Kumar. Provable systemwide safety in intelligent intersections. IEEE Transactions on Vehicular Technology, 60(3):804-818, March 2011.

[9] N. Murgovski, L. M. Johannesson, and B. Egardt. Optimal Battery Dimensioning and Control of a CVT PHEV Powertrain. IEEE Transactions on Vehicular Technology, 63(5):2151-2161, Jun 2014.

[10] R. Quirynen, M. Vukov, M. Zanon, and M. Diehl. Autogenerating Microsecond Solvers for Nonlinear MPC: a Tutorial Using ACADO Integrators. Optimal Control Applications and Methods, 36:685-704, 2014.

[11] M. Zanon and T. Faulwasser. Economic MPC without terminal constraints: Gradient-correcting end penalties enforce asymptotic stability. Journal of Process Control, 63:1 - 14, 2018.

[12] M. Zanon, S. Gros, and M. Diehl. Indefinite Linear MPC and Approximated Economic MPC for Nonlinear Systems. Journal of Process Control, 24:1273-1281, 2014.

[13] M. Zanon, S. Gros, and M. Diehl. A Tracking MPC Formulation that is Locally Equivalent to Economic MPC. Journal of Process Control, 2016.

[14] M. Zanon, S. Gros, and M. Diehl. A Periodic Tracking MPC that is Locally Equivalent to Periodic Economic MPC. In Proceedings of the 2017 IFAC World Congress, 2017. (submitted).

[15] M. Zanon, S. Gros, H. Wymeersch, and P. Falcone. An Asynchronous Algorithm for Optimal Vehicle Coordination at Traffic Intersections. In 20th IFAC World Congress, 2017.

[16] M. Zanon, R. Hult, S. Gros, and P. Falcone. Experimental Validation of Distributed Optimal Vehicle Coordination. In Proceedings of the European Control Conference, 2018.

[17] Y. J. Zhang, A. A. Malikopoulos, and C. G. Cassandras. Optimal control and coordination of connected and automated vehicles at urban traffic intersections. In 2016 American Control Conference (ACC). 